\title{
Polyguanamine Derivative-Based Supramolecular Assemblies with Multiple Hydrogen Bonding and Their Metal Scavenging Abilities
}

\author{
Keito Fukushi, ${ }^{a}$ Haruka Maruyama, ${ }^{\mathrm{b}}$ Yuji Shibasaki, ${ }^{\mathrm{c}}$ and Atsuhiro Fujimori ${ }^{\mathrm{a},}{ }^{*}$ \\ ${ }^{a}$ Graduate School of Science and Engineering, and ${ }^{b}$ Faculty of Engineering, Saitama \\ University, 255 Shimo-okubo, Sakura-ku, Saitama 338-8570, Japan \\ ${ }^{c}$ Department of Chemistry \& Biological Sciences, Faculty of Science \& Engineering, Iwate \\ University, 4-3-5 Ueda, Morioka, Iwate 020-8551, Japan \\ *Corresponding author \\ Tel. and Fax: $+81-48-858-3503$ \\ E-mail address: fujimori@apc.saitama-u.ac.jp
}




\section{[Figure Caption]}

Figure S1. Schematic illustration of interfacial conformation as (a) flat-on, (b) edge-on, and (c) end-on.

Figure S2. Low angle out-of-plane XRD profiles of LB multilayers of polyguanamine derivatives ((a) PsTC-ODA, (b) PsTC-BAFL, and (c) PsTC- $m$ PDA).

Figure S3. Schematic illustrations of the abundant negative electric field in the annulus and the corresponding sizes.

Figure S4. Details of the desorption experiment of scavenged metal ions and its corresponding data. And evaluation of recycling ability by re-immersion of metal desorption LB film.

Figure S5. In-plane XRD profiles of LB multilayers of three kinds of polyguanamine derivatives before and after $\mathrm{Cd}^{2+}$ ion scavenging.

Figure S6. AFM images of monolayer of polyguanamine derivatives before and after metal scavenging.

Figure S7. Summarized results of this study. 


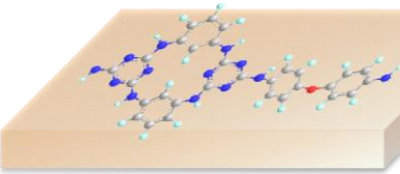

(a) Flat-on

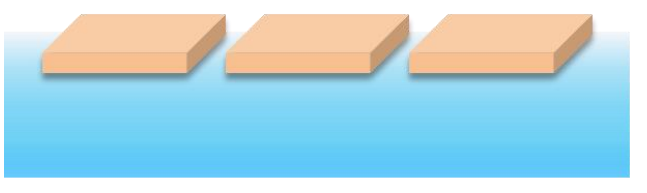

(b) Edge-on

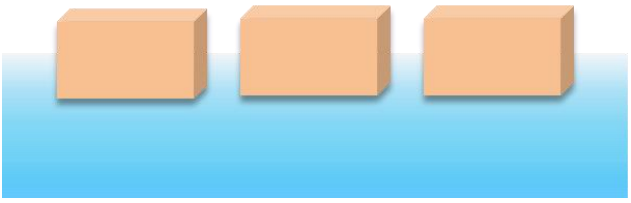

(c) End-on

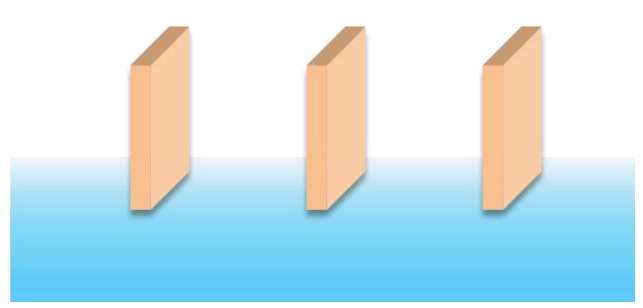

Figure S1

K. Fukushi, et al 


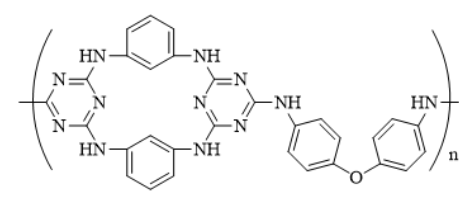

(a)

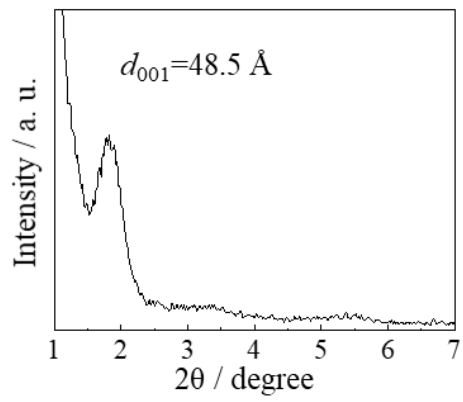

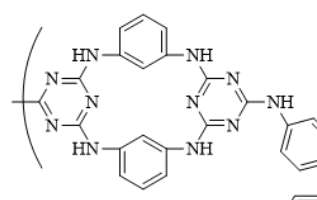

(b)

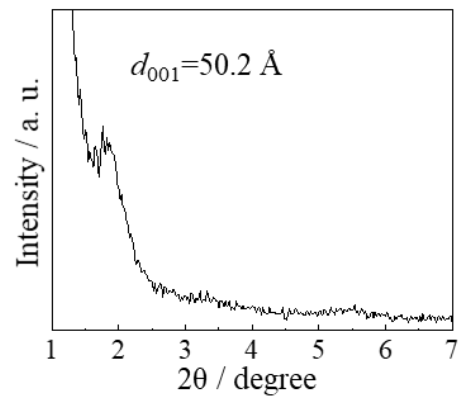

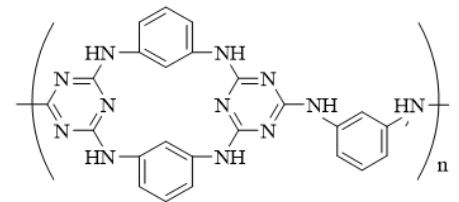

(c)

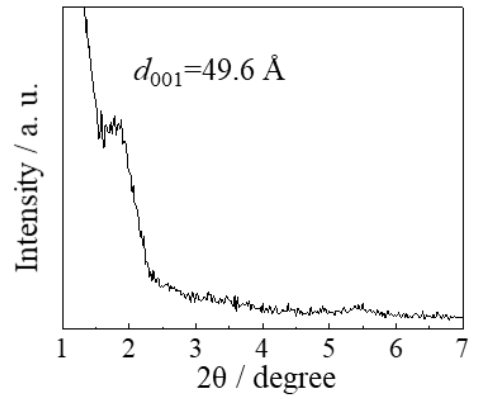

Figure S2

K. Fukushi, et al 

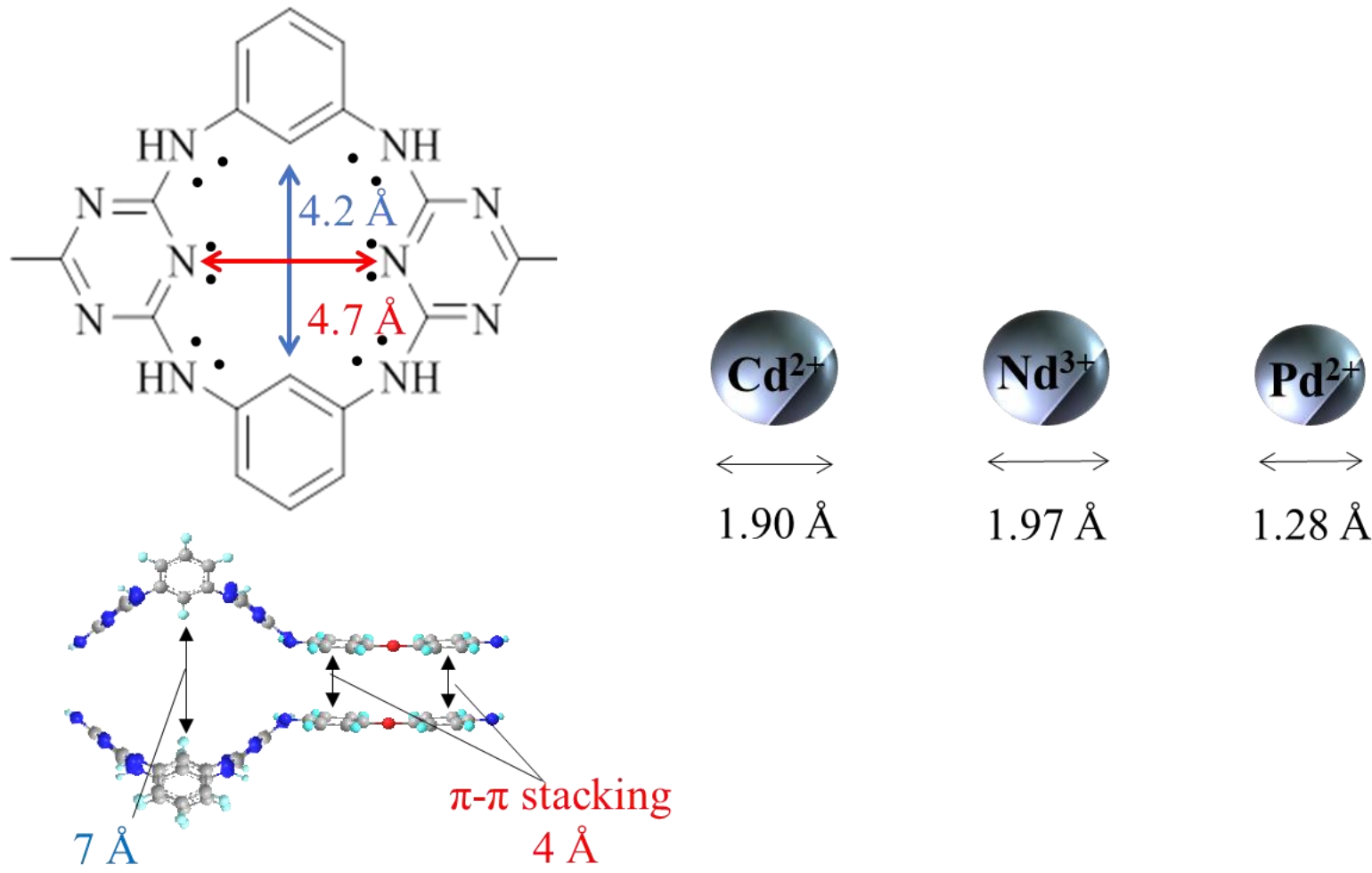

$1.90 \AA$

$1.97 \AA$

$1.28 \AA$

Figure S3

K. Fukushi, et al 


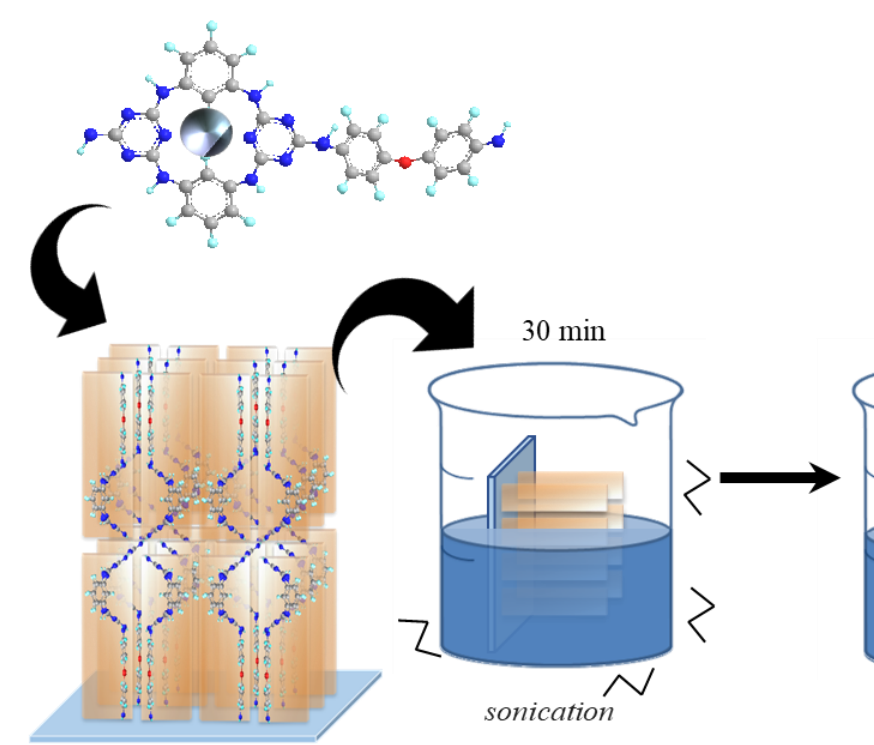

Sonication time $30 \mathrm{~min}$
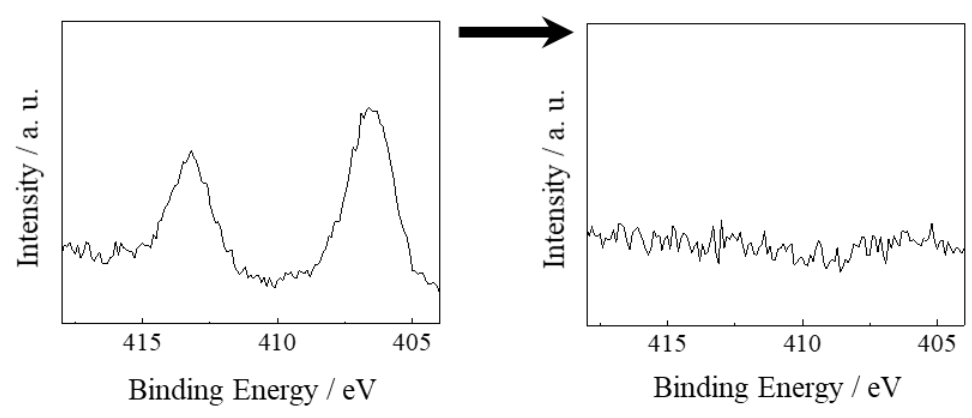

Re-immersing

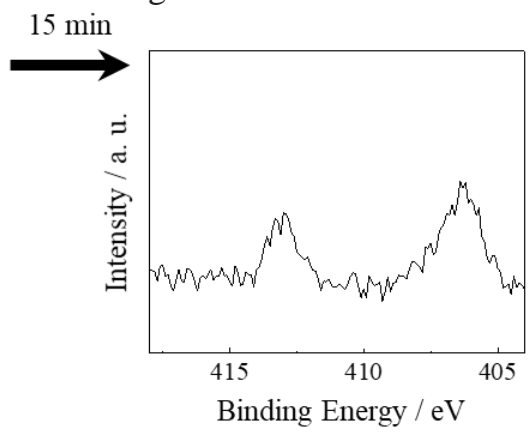

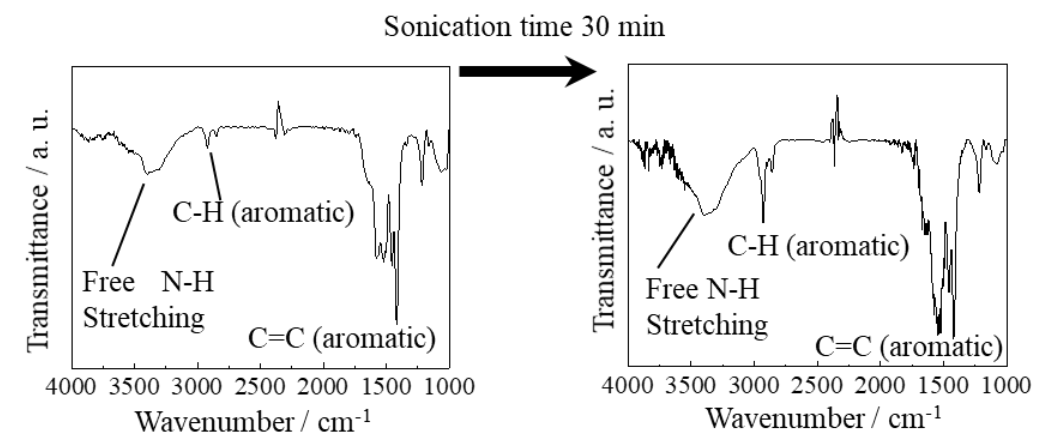

Figure S4

K. Fukushi, et al 

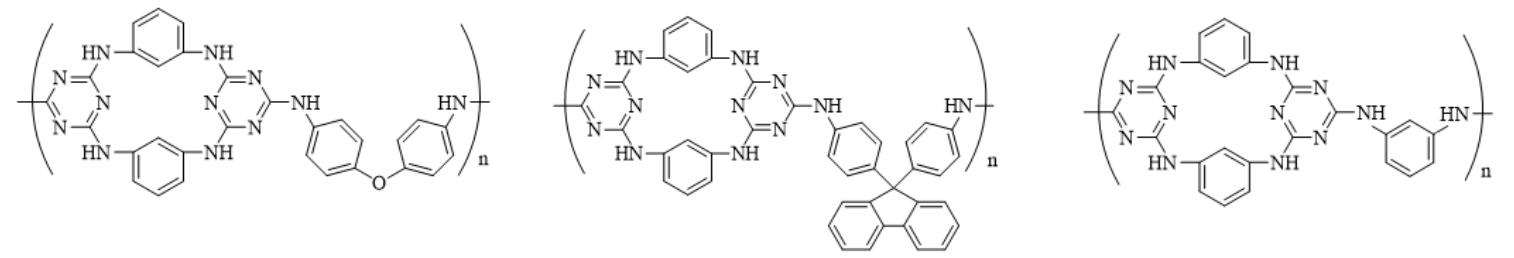

- Metal free

$-\mathrm{Cd}^{2+}$ complex

(a)

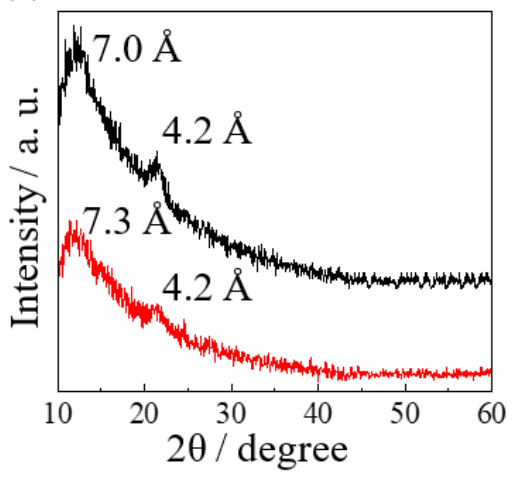

(b)

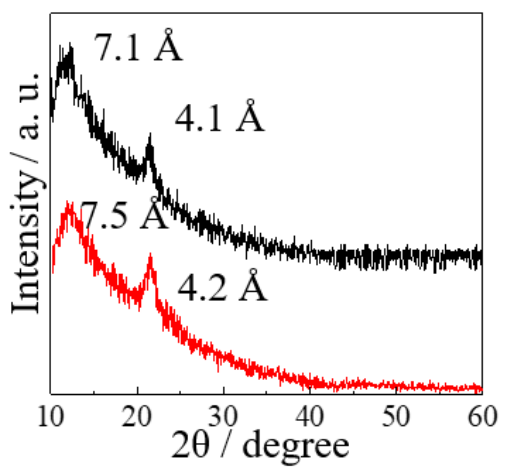

(c)

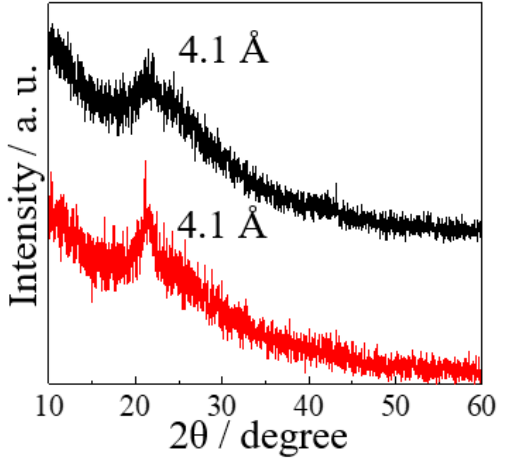

Figure S5

K. Fukushi, et al 


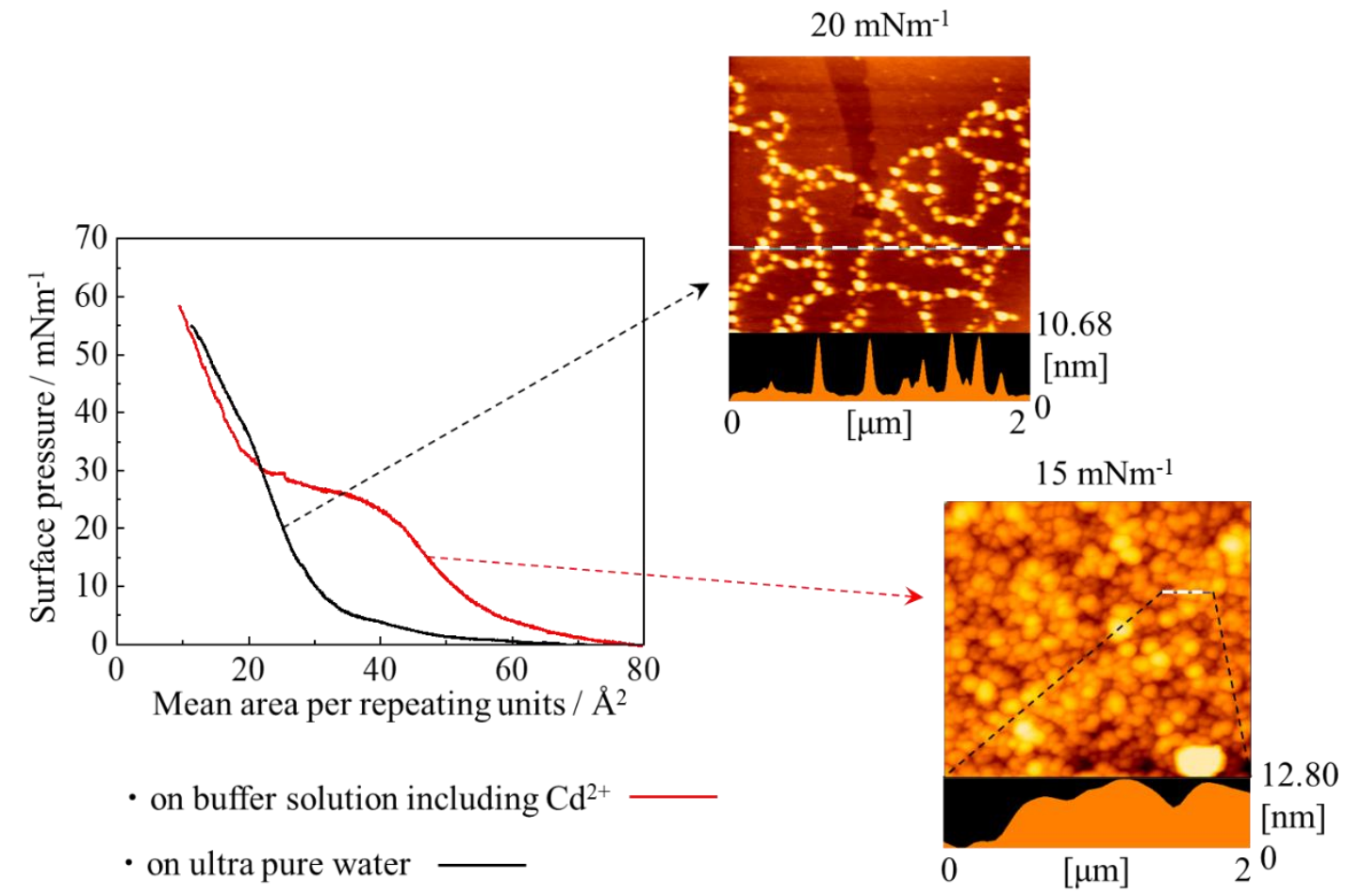

Figure S6

K. Fukushi, et al 


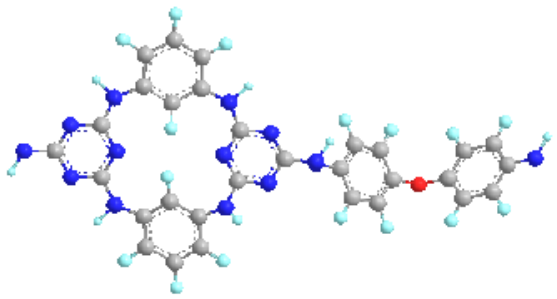

Polymers with cyclic parts

Monolayer Spreading

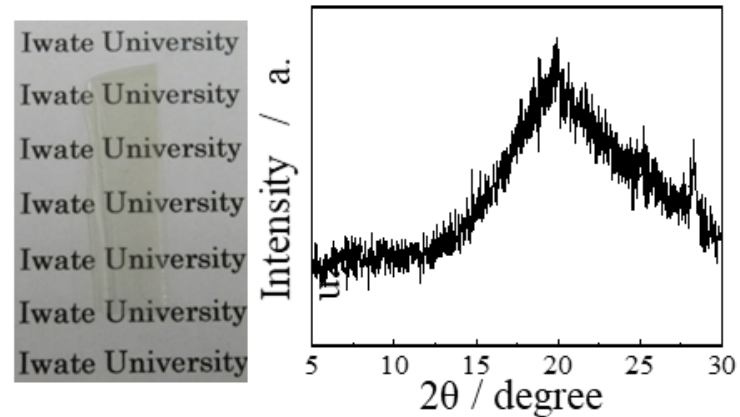

Amorphous in bulk state

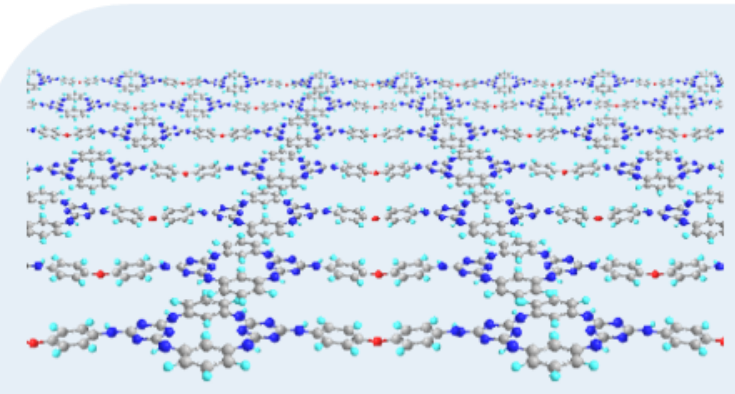

Supramolecular structure
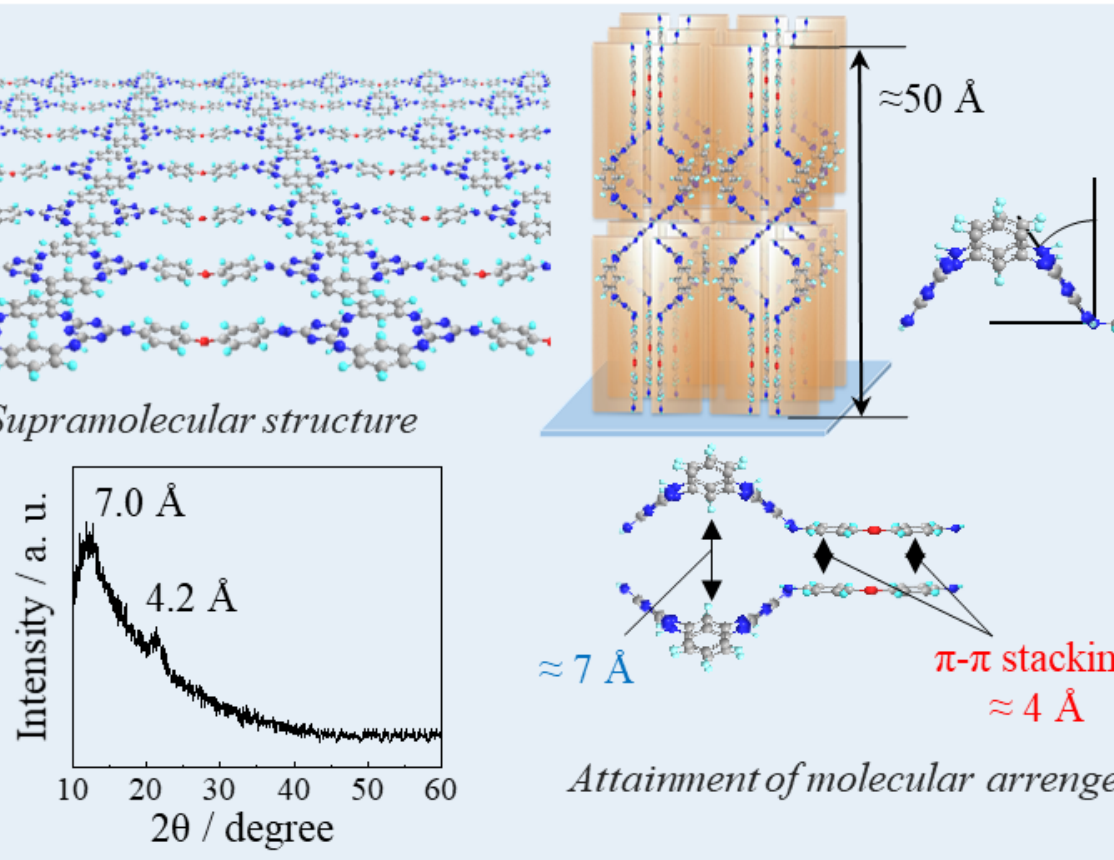

$42^{\circ}$

$\approx 7 \AA$
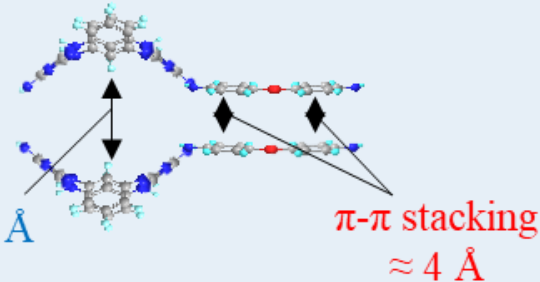

Attainment of molecular arrengement

Metal
scavenging $\mathrm{Cd}^{2+} \mathrm{Nd}^{3+} \mathrm{Pd}^{2+}$
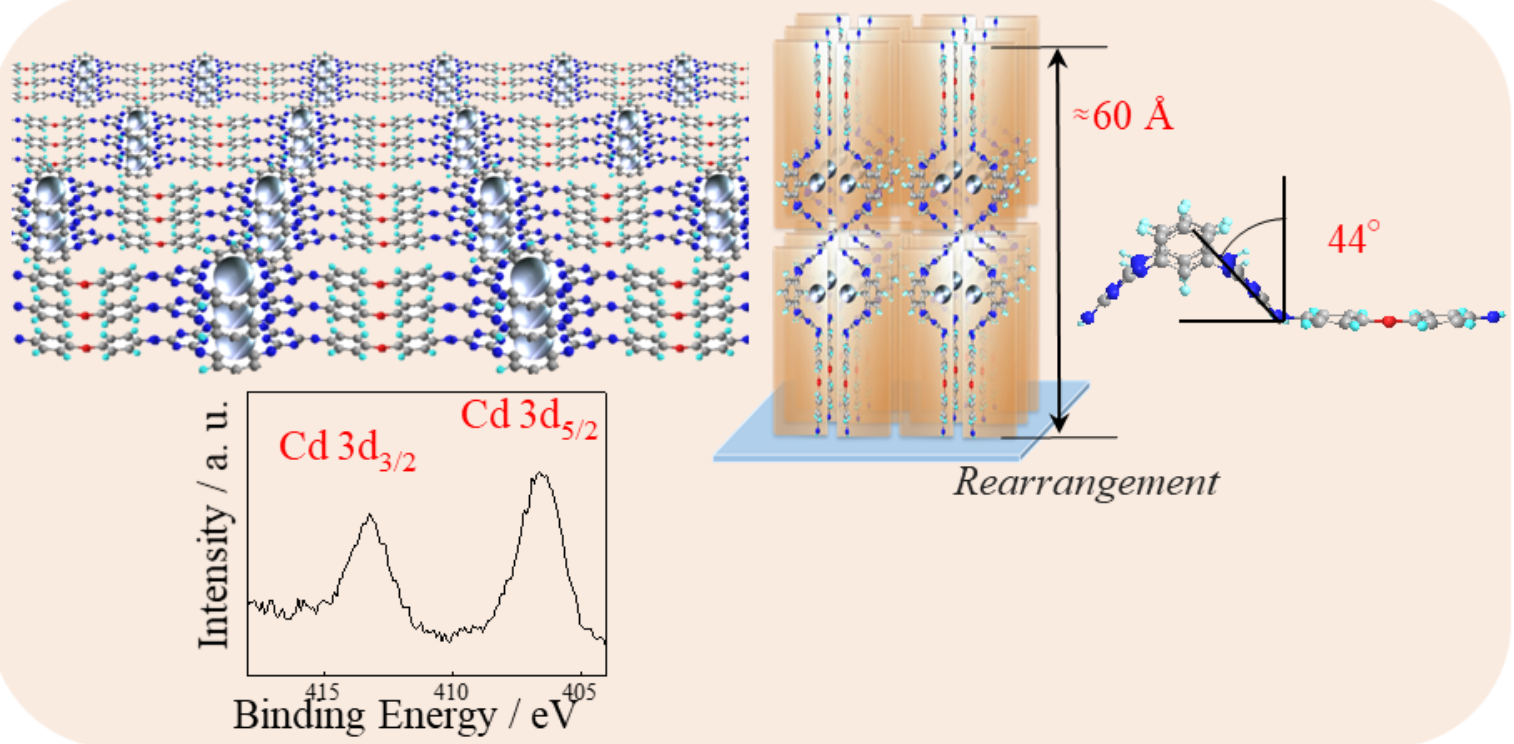

Figure S7

K. Fukushi, et al 